\title{
On Contraction of Lie Algebra Representations
}

\author{
U. Cattaneo ${ }^{1 \star}$ and W. Wreszinski ${ }^{2 \star \star}$ \\ 1 Institut de Physique, Université de Neuchâtel, CH-2000 Neuchâtel, Switzerland \\ 2 Seminar für theoretische Physik, ETH Hönggerberg, CH-8093 Zürich, Switzerland
}

\begin{abstract}
Given a net $\left(\mathfrak{g}_{\imath}\right)$ of finite-dimensional real Lie algebras contracting into a Lie algebra $\hat{\mathrm{g}}$, a representation $\hat{\pi}_{J}$ of $\hat{g}$ is constructed explicitly as "limit" of a net $\left(\pi_{t}\right)$ of representations, each $\pi_{t}$ being a representation of $\mathfrak{g}_{t}$ on a complex Hilbert space $\mathfrak{H}_{l}$. Conditions are imposed on the net $\left(\pi_{\imath}\right)$ implying that the carrier space of $\hat{\pi}_{J}$ contain a $\hat{\pi}_{J}(\hat{\mathfrak{g}})$-stable set of vectors which are analytic for all $\hat{\pi}_{J}(g)(g \in \mathscr{G})$, where $\mathscr{G}$ is a basis of $\hat{\mathrm{g}}$. As a corollary, the corresponding result for contractions of representations of simply connected finitedimensional real Lie groups is derived.
\end{abstract}

\section{Introduction}

In this note, we present a theory of contraction of nets of Lie algebra representations. Let $J$ be a directed system (usually a subset of $\mathbf{R}$ with the induced ordering) and, for each $\imath \in J$, let $\pi_{\iota}$ be a representation of a finite-dimensional real Lie algebra $\mathfrak{g}_{\imath}$ on a complex Hilbert space $\mathfrak{H}_{l}$. Suppose that, in addition, every $\mathfrak{g}_{\imath}$ is isomorphic to a reference Lie algebra $\mathfrak{g}$ which is "contracting into $\hat{g}$ " in a precise sense reviewed in Sect. II.2. We define and investigate a representation $\hat{\pi}_{J}$ of the contracted Lie algebra $\hat{\mathfrak{g}}$ whose carrier space is constructed in terms of the net $\left(\mathfrak{G}_{l}\right)$. The adopted definition permits to give the operators of $\hat{\pi}_{J}$ directly, without appealing to matrix elements, in a way which seems naturally suited to the problem considered. Since the theory of Lie algebra contraction is rooted in a notion of limit, which is responsible for the fact that $\mathfrak{g}$ and $\hat{g}$ are not, in general, isomorphic, the final space cannot be defined in a "canonical" way [for instance, as the Hilbert sum or as a tensor product of the family $\left.\left(\mathfrak{H}_{2}\right)\right]$. Our definition is rather more similar in spirit to Trotter's definition of a Banach space approximated by a sequence of Banach spaces ([1], Sect. 2), with the main difference that Trotter presupposes knowledge of the final space.

* Supported by the Swiss National Science Foundation

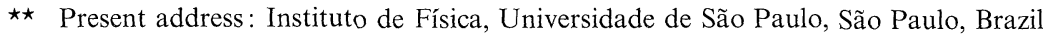


More precisely, the paper is organized as follows. In Sect. II.1, we define the "limit" $\mathfrak{G}_{J}\left(\right.$ resp. $\left.A_{J}^{F}\right)$ of a net $\left(\mathfrak{H}_{l}\right)$ [resp. $\left.\left(A_{l}\right)\right]$ of complex Hilbert spaces [resp. of operators in $\left.\left(\mathfrak{H}_{1}\right)\right]$ indexed by a directed system $J$. We further provide conditions sufficient to assure the nontriviality of the operator $A_{J}^{F}$ in $\mathfrak{S}_{J}$ (Proposition 1). In Proposition 2, conditions are imposed, for each $\imath \in J$, on the action of an operator $A_{\imath}$ on a set $\mathfrak{S}_{\imath}$ of vectors of $\mathfrak{H}_{l}$ in order that the net $\left(A_{\imath}\right)$ should define a nontrivial operator $A_{J}^{F}$ in $\mathfrak{S}_{J}$ and that the linear span of the "limit vectors" of the net $\left(\mathfrak{S}_{l}\right)$ should be a set of analytic vectors for $A_{J}^{F}$. In Sect. II.2, we remind the notion of contraction of a net $\left(\mathfrak{g}_{l}\right)$ of finite-dimensional real Lie algebras into a Lie algebra $\hat{\mathfrak{g}}$ [2-5]. We also introduce the concept of a contracting net $\left(\pi_{2}\right)$ of representations of $\left(\mathfrak{g}_{l}\right)$ on $\left(\mathfrak{G}_{l}\right)$ and, in Proposition 3 , we prove that a contracting net $\left(\pi_{l}\right)$ always defines a representation $\hat{\pi}_{J}$ of $\hat{\mathfrak{g}}$. In Proposition 4 , we show the existence of $\hat{\pi}_{J}$ defined by a net $\left(\pi_{l}\right)$ satisfying conditions which are essentially borrowed from the assumptions of Proposition 2. Moreover, we prove that there exists a $\hat{\pi}_{J}(\hat{\mathfrak{g}})$-stable set of vectors which are analytic for all $\hat{\pi}_{J}(g)$ with $g$ belonging to an appropriate basis of $\hat{\mathfrak{g}}$. From this it follows that our assertion on the existence of $\hat{\pi}_{J}$ in Proposition 4 has a counterpart in a result on Lie group representations. This is stated in a corollary at the end of Sect. II.2.

In what follows, $J$ will always denote a directed system and every net considered in the present paper will be indexed by $J$; we shall always write $\left(\mathfrak{G}_{l}\right)$, $\left(A_{\imath}\right), \ldots$ short for $\left(\mathfrak{H}_{\imath}\right)_{l \in J},\left(A_{\imath}\right)_{\imath \in J}, \ldots$ The symbol $\mathrm{D}(A)$ will denote the domain of a (bounded or unbounded linear) operator $A$ and, given a subset $D$ of a Hilbert space $\mathfrak{H}, \operatorname{sp}(D)$ will stand for the linear span of $D$ in $\mathfrak{H}$.

\section{Contraction of Representations}

\section{II.1. Hilbert Spaces of Equivalence Classes of Convergent Nets}

For each $\imath \in J$, let $\mathfrak{H}_{\imath}$ be a complex Hilbert space and let $(\cdot \mid \cdot)_{\imath}$ be the inner multiplication on $\mathfrak{H}_{l}$. We say that an element $\left(\phi_{l}\right)$ of the product vector space $\prod_{l} \mathfrak{H}_{l}$ of the family $\left(\mathfrak{G}_{l}\right)$ is convergent if the net $\left(\left\|\phi_{\imath}\right\|_{l}\right)$ converges in $\mathbf{R}$. Let $\mathscr{H}_{J}$ be the vector subspace of all convergent nets $\left(\phi_{l}\right) \in \prod \mathfrak{H}_{\imath}$ equipped with the positive Hermitian form $(\cdot \mid \cdot)_{J}$ defined by

$$
\left(\left(\phi_{\imath}\right) \mid\left(\phi_{\imath}^{\prime}\right)\right)_{J}=\lim _{\imath}\left(\phi_{\imath} \mid \phi_{\imath}^{\prime}\right)_{\imath} \text {. }
$$

We note that (II.1) is meaningful by reason of the polarization identity. If $\mathscr{J}$ is the vector subspace of all nets $\left(\phi_{l}\right) \in \mathscr{H}_{J}$ such that $\left\|\left(\phi_{l}\right)\right\|_{J}=0$, we define a Hilbert space $\mathfrak{H}_{J}$ as the completion of the quotient vector space $\mathscr{H}_{J} / \mathscr{J}$ endowed with the extended quotient form which we shall also write $(\cdot \mid \cdot)_{J}$.

We shall denote by $\left[\phi_{l}\right],\left[\psi_{l}\right], \ldots$, respectively, the equivalence classes modulo $\mathscr{J}$ of the elements $\left(\phi_{\imath}\right),\left(\psi_{\imath}\right), \ldots$ of $\mathscr{H}_{J}$. If $D$ is a subset of $\mathscr{H}_{J}$, the symbol $[D]$ will stand for the subset of all $\left[\phi_{l}\right] \in \mathfrak{H}_{J}$ with $\left(\phi_{\imath}\right) \in D$.

Notice that if $\left(\phi_{l}\right) \in \mathscr{H}_{J}$ and if $\left(\gamma_{l}\right)$ is a net of complex numbers converging to $\gamma$ in C, then $\left[\gamma_{l} \phi_{l}\right]=\gamma\left[\phi_{l}\right]$.

Remark 1. If there exists a complex Hilbert space $\mathfrak{H}$ such that the net $\left(\mathfrak{H}_{l}\right)$ satisfies $\mathfrak{H}_{\imath}=\mathfrak{H}$ for all $\imath \in J$, then the mapping $\alpha: \phi \mapsto\left[\phi_{l}\right]$ with $\phi_{\imath} \approx \phi$ for all $\iota \in J$ is a closed 
injective homomorphism of $\mathfrak{H}$ into $\mathfrak{H}_{J}$. However, in general, $\alpha(\mathfrak{H})$ is a proper subspace of $\mathfrak{H}_{J}$. For instance, if $J=\mathbf{N}$ and $\mathfrak{H}$ is separable with an orthonormal basis $\left\{\phi^{(n)}\right\}_{n \in \mathbf{N}}$, then the element $\left[\phi_{n}\right]$ of $\mathfrak{H}_{J}$ defined by $\phi_{n}=\phi^{(n)}(n \in \mathbf{N})$ is orthogonal to $\alpha(\mathfrak{H})$.

A net of operators in $\left(\mathfrak{S}_{l}\right)$ is a family $\left(A_{\imath}\right)$ of (linear) operators, where every $A_{\imath}$ is an operator in $\mathfrak{S}_{l}$. Let $D_{J}\left(A_{\imath}\right)$ be the vector subspace of all $\left(\phi_{\imath}\right) \in \mathscr{H}_{J}$, with $\phi_{\imath} \in \mathrm{D}\left(A_{\imath}\right)$ for all $\imath \in J$, such that the net $\left(A_{\imath} \phi_{l}\right)$ is convergent and, given an arbitrary vector subspace $F$ of $D_{J}\left(A_{\imath}\right)$, let $D_{u}^{F}\left(A_{\imath}\right)$ be the vector subspace of all $\left(\phi_{\imath}\right) \in F$ such that we have $\left[A_{\imath} \phi_{l}\right]=\left[A_{l} \phi_{l}^{\prime}\right]$ whenever $\left(\phi_{l}^{\prime}\right)$ is an element of $F$ in the equivalence class of $\left(\phi_{l}\right)$ modulo $\mathscr{J}$. Then we define an operator $A_{J}^{F}$ in $\mathfrak{H}_{J}$ by

$$
A_{J}^{F}\left[\phi_{\imath}\right]=\left[A_{\imath} \phi_{\imath}\right] \quad\left(\left(\phi_{\imath}\right) \in D_{u}^{F}\left(A_{\imath}\right)\right)
$$

with $\mathrm{D}\left(A_{J}^{F}\right)=\left[D_{u}^{F}\left(A_{\imath}\right)\right]$. It can happen that $\mathrm{D}\left(A_{J}^{F}\right)=\{0\}$, i.e., that $A_{J}^{F}$ is trivial. If $F^{\prime}$ is a vector subspace of $F$, then $A_{J}^{F^{\prime}} \subseteq A_{J}^{F}$, i.e., $\mathrm{D}\left(A_{J}^{F^{\prime}}\right) \subseteq \mathrm{D}\left(A_{J}^{F}\right)$ and $A_{J}^{F^{\prime}}\left[\phi_{l}\right]=A_{J}^{F}\left[\phi_{l}\right]$ for all $\left[\phi_{l}\right] \in \mathrm{D}\left(A_{J}^{F^{\prime}}\right)$ (in words: $A_{J}^{F^{\prime}}$ is a restriction of $A_{J}^{F}$ ).

Remark 2. Even if the elements $\left(\phi_{l}\right)$ and $\left(\phi_{l}^{\prime}\right)$ of $\mathscr{H}_{J}$ are in the same equivalence class modulo $\mathscr{J}$, it can occur that $\left(A_{\imath} \phi_{l}\right)$ is convergent but $\left(A_{1} \phi_{1}^{\prime}\right)$ is not.

Remark 3. The above definitions are related to those introduced, in another context, by Trotter [1] and Kurtz [6]. For each $\imath \in J$, we can define a linear mapping $P_{\imath}$ of $\mathscr{H}_{J} / \mathscr{J}$ into $\mathfrak{H}_{l}$ such that

$$
\lim _{l}\left\|P_{l}\left[\phi_{i^{\prime}}\right]\right\|_{l}=\left\|\left[\phi_{i^{\prime}}\right]\right\|_{J}
$$

for all $\left(\phi_{l^{\prime}}\right) \in \mathscr{H}_{J}$. But, contrarily to the corresponding Trotter's mappings, the $P_{\imath}$ are, in general, unbounded [see however Proposition 4(v)].

Of particular importance in the sequel will be nets $\left(A_{l}\right)$ of operators in $\left(\mathfrak{H}_{l}\right)$ satisfying the following condition have

(K) For each $l \in J$, each $\phi \in \mathrm{D}\left(A_{\imath}\right)$, and each real (resp. imaginary) scalar $\lambda$, we

$$
\left\|\lambda \phi-A_{\imath} \phi\right\|_{\imath} \geqq|\lambda|\|\phi\|_{\imath} .
$$

Notice that nets of skew-symmetric (resp. symmetric) operators satisfy Condition $(\mathrm{K})$, as can be checked by developing the square of the left-hand side of (II.3).

Proposition 1. Let $\left(A_{l}\right)$ be a net of operators in $\left(\mathfrak{H}_{l}\right)$ satisfying Condition $(\mathrm{K})$. If $F$ is a vector subspace of $D_{J}\left(A_{\imath}\right) \cap D_{J}\left(A_{\imath}^{2}\right)$, then $D_{u}^{F}\left(A_{\imath}\right)=F$.

Proof. Our proof is modeled on that of Kurtz's Lemma (1.1) [6]. Let $\left(\phi_{\imath}\right),\left(\phi_{\imath}^{\prime}\right)$ be two elements of $F$ in the same equivalence class modulo $\mathscr{J}$ and put $\left(\psi_{\imath}\right)=\left(\phi_{\imath}\right)-\left(\phi_{l}^{\prime}\right)$. Then, if $\lambda$ is any real (resp. imaginary) scalar, we have by virtue of (II.3)

$$
\lim _{\imath}\left\|\left\{\lambda \operatorname{Id}_{\mathfrak{H}_{l}}-A_{\imath}\right\}\left\{A_{\imath} \psi_{\imath}+\lambda \psi_{\imath}\right\}\right\|_{\imath}=\left\|\left[A_{l}^{2} \psi_{l}\right]\right\|_{J} \geqq|\lambda|\left\|\left[A_{\imath} \psi_{l}\right]\right\|_{J},
$$

whence $\left[A_{\imath} \psi_{\imath}\right]=0$. 
Let $\left(A_{\imath}\right),\left(B_{\imath}\right)$ be two nets of operators both in $\left(\mathfrak{H}_{\imath}\right)$ and let $F$ be a vector subspace of $D_{J}\left(A_{l} B_{l}\right)$ such that $\left(A_{l} \phi_{l}\right) \in F,\left(B_{l} \phi_{l}\right) \in F$ whenever $\left(\phi_{l}\right) \in F$. Then we define an operator $(A B)_{J}^{F}$ in $\mathfrak{H}_{J}$ by

$$
(A B)_{J}^{F}\left[\phi_{l}\right]=\left[A_{l} B_{l} \phi_{l}\right] \quad\left(\left(\phi_{l}\right) \in D_{u}^{F}\left(A_{l} B_{\imath}\right)\right)
$$

with $\mathrm{D}\left((A B)_{J}^{F}\right)=\left[D_{u}^{F}\left(A_{\imath} B_{l}\right)\right]$.

Remark 4. If $F$ is as in the definition of $(A B)_{J}^{F}$ and if, in addition, $D_{u}^{F}\left(A_{\imath}\right)$ $=D_{u}^{F}\left(B_{\imath}\right)=F$, we have $D_{u}^{F}\left(A_{\imath} B_{\imath}\right)=F$ and $(A B)_{J}^{F}=A_{J}^{F} B_{J}^{F}$.

Proposition 2. Let $\left(A_{t}\right)$ be a net of operators in $\left(\mathfrak{H}_{1}\right)$ satisfying Condition $(\mathrm{K})$, let $S$ be a subset of $\mathbf{R}$, and let $\mathfrak{\Xi}_{J}=\left\{\left[\phi_{l}^{(s)}\right]\right\}_{s \in S}$ be a norm-bounded subset of $\mathfrak{H}_{J}$ with $\phi_{\imath}^{(s)} \in \mathrm{D}\left(A_{\imath}\right)$ for all $\imath \in J$ and all $s \in S$. Suppose that, for each $\imath \in J$ and each $s \in S$, we have

$$
A_{\imath} \phi_{l}^{(s)}=\sum_{m=-k}^{k} c_{l, s, m} \phi_{l}^{(s+m)},
$$

where $k$ is a positive integer, the $c_{l, s, m}$ are complex numbers with $c_{l, s, m}=0$ whenever $s+m \notin S$, and, for any fixed pair $s, m$, the net $\left(c_{l, s, m}\right)$ converges in $\mathbf{C}$ to $c_{s, m}$. If there exist two real numbers $v$ and $t$ such that $\left|c_{s, m}\right| \leqq v(|s|+|t|)$ for $-k \leqq m \leqq k$ and all $s \in S$, then

(i) $\operatorname{sp}\left(\left\{\left(\phi_{\imath}^{(s)}\right)\right\}_{s \in S}\right)$ is a vector subspace of $W=\bigcap_{n \in \mathbf{N}^{k}} D_{J}\left(A_{\imath}^{n}\right)$.

(ii) $\operatorname{sp}\left(\Theta_{J}\right)$ is a set of analytic vectors for $A_{J}^{F}$, where $F$ is any vector subspace of $W$ containing $\operatorname{sp}\left(\left\{\left(\phi_{l}^{(s)}\right)\right\}_{s \in S}\right)$.

Proof. Assertion (i) follows from (II.4). To prove (ii), we remark that we have $D_{u}^{F}\left(A_{\imath}^{n}\right)=D_{u}^{F}\left(A_{\imath}\right)=F$ for all $n \in \mathbf{N}^{*}$ by virtue of Proposition 1 . Hence $\operatorname{sp}\left(\Im_{J}\right)$ is a set of $C^{\infty}$-vectors for $A_{J}^{F}$ because $\left(A^{n}\right)_{J}^{F}=\left(A_{J}^{F}\right)^{n}$ for all $n \in \mathbf{N}^{*}$. Now let $r$ be a real number $\geqq 1$ such that $\left\|\left[\phi_{l}^{(s)}\right]\right\|_{J} \leqq r$ for all $s \in S$. For each $n \in \mathbf{N}^{*}$ and each $s \in S$, we have (with $-k \leqq m_{i} \leqq k$ and $\left.1 \leqq i \leqq n\right)$

$$
\begin{aligned}
& \left\|\left(A_{J}^{F}\right)^{n}\left[\phi_{l}^{(s)}\right]\right\|_{J} \\
& \quad=\lim _{l}\left\|\sum_{m_{l}} c_{l, s, m_{1}} c_{l, s+m_{1}, m_{2}} \ldots c_{l, s+m_{1}+\ldots+m_{n-1}, m_{n}} \phi_{l}^{\left(s+m_{1}+m_{2}+\ldots+m_{n}\right)}\right\|_{l} \\
& \quad \leqq \sum_{m_{l}}\left|c_{s, m_{1}}\right| \ldots\left|c_{s+m_{1}+\ldots+m_{n-1}, m_{n}}\right| r \\
& \quad \leqq(2 k+1)^{n} v^{n}(|s|+|t|)(|s|+|t|+k) \ldots(|s|+|t|+(n-1) k) r \leqq d^{n} n !
\end{aligned}
$$

where $d$ is some positive real number depending on $k, r, v$, and $(|s|+|t|)$, but not on $n$. Therefore $\left[\phi_{l}^{(s)}\right]$ is an analytic vector for $A_{J}^{F}$ and $\operatorname{sp}\left(\Theta_{J}\right)$ is a set of analytic vectors for $A_{J}^{F}$.

\section{II.2. Contraction of Nets of Lie Algebra Representations}

To begin with, let us recollect some facts about contraction of Lie algebras. In this Section, $V$ will always stand for a finite-dimensional real vector space.

Let $\mathfrak{M}_{V}$ be the algebraic set of all Lie multiplications on $V$, i.e., the set of all bilinear alternating mappings of $V \times V$ into $V$ satisfying the Jacobi identity. Notice that, by choosing a basis of $V$, we can identify $\mathfrak{M}_{V}$ with the set of all families of 
structure constants of Lie algebras with underlying vector space $V$. We give $\mathfrak{M}_{V}$ the topology induced by the canonical Hausdorff topology of the vector space of all bilinear alternating mappings of $V \times V$ into $V$. We shall denote by alg $(V, \mu)$ the Lie algebra with underlying vector space $V$ and Lie multiplication $\mu$.

A net $\left(\operatorname{alg}\left(V, \mu_{t}\right)\right)$ of Lie algebras is said to be contracting with respect to a Lie algebra $\operatorname{alg}(V, \mu)$ if all its elements are isomorphic to $\operatorname{alg}(V, \mu)$ and if the net $\left(\mu_{\imath}\right)$ converges in $\mathfrak{M}_{V}$ to some Lie multiplication $\hat{\mu}$. In other words, the net $\left(\operatorname{alg}\left(V, \mu_{\imath}\right)\right)$ is contracting into alg $(V, \hat{\mu})$ if there exists a net $\left(\Gamma_{t}\right)$ of automorphisms of $V$ such that, for each $t \in J$, the mapping $\Gamma_{l}$ is an isomorphism of $\operatorname{alg}\left(V, \mu_{l}\right)$ onto $\operatorname{alg}(V, \mu)$ and

$$
\lim _{\imath} \Gamma_{\imath}^{-1}\left(\mu\left(\Gamma_{\imath}(g), \Gamma_{\imath}\left(g^{\prime}\right)\right)\right)=\hat{\mu}\left(g, g^{\prime}\right)
$$

for all $g, g^{\prime}$ in $V$. Alternatively, if we are given the net $\left(\Gamma_{\imath}\right)$, then the net $\left(\mu_{\imath}\right)$ is defined by

$$
\mu_{\imath}\left(g, g^{\prime}\right)=\Gamma_{\imath}^{-1}\left(\mu\left(\Gamma_{\imath}(g), \Gamma_{\imath}\left(g^{\prime}\right)\right)\right) .
$$

The Lie algebra $\operatorname{alg}(V, \hat{\mu})$ is said to be the contracted Lie algebra of the net $\left(\operatorname{alg}\left(V, \mu_{l}\right)\right)$ and the operation performed is called a contraction (of a net of Lie algebras). By abuse of language, we shall also say that $\operatorname{alg}(V, \hat{\mu})$ is a contraction of $\operatorname{alg}(V, \mu)$. We shall call alg $(V, \mu)$ the reference Lie algebra and $\left(\Gamma_{\imath}\right)$ the reference net of automorphisms of $V$. Notice that we do not exclude the trivial case of a contracted Lie algebra isomorphic to the reference Lie algebra.

We remind that a representation of a Lie algebra $\mathfrak{g}=\operatorname{alg}(V, \mu)$ on a complex Hilbert space $\mathfrak{H}$ is an ordered pair $(\pi, D(\pi))$, where $\pi$ is a mapping of $\mathfrak{g}$ into the set of all operators in $\mathfrak{H}$ and $D(\pi)$ is a $\pi(\mathfrak{g})$-stable vector subspace of $\bigcap_{g \in \mathfrak{g}} D(\pi(g))$ dense in $\mathfrak{S}$, such that

$$
\begin{aligned}
& \pi\left(\gamma g+\gamma^{\prime} g^{\prime}\right) \phi=\gamma \pi(g) \phi+\gamma^{\prime} \pi\left(g^{\prime}\right) \phi, \\
& \left(\pi(g) \pi\left(g^{\prime}\right)-\pi\left(g^{\prime}\right) \pi(g)\right) \phi=\pi\left(\mu\left(g, g^{\prime}\right)\right) \phi
\end{aligned}
$$

for all $\gamma, \gamma^{\prime}$ in $\mathbf{R}$, all $g, g^{\prime}$ in $\mathfrak{g}$, and all $\phi \in \mathrm{D}(\pi)$. In what follows, we shall simply say "the representation $\pi$ ", tacitly understanding that $\mathrm{D}(\pi)$, which is called the domain of $\pi$, is also given. By the restriction of $\pi$ to a $\pi(\mathfrak{g})$-stable vector subspace $D^{\prime}$ of $\mathrm{D}(\pi)$, we shall mean the representation $\pi^{\prime}$ of $\mathfrak{g}$ on the closure of $D^{\prime}$ in $\mathfrak{S}$ defined by $\pi^{\prime}(g)\left|D^{\prime}=\pi(g)\right| D^{\prime}$ and with $\mathrm{D}\left(\pi^{\prime}\right)=D^{\prime}$. We shall denote by Env $(\mathrm{g})$ the enveloping algebra of $\mathfrak{g}$; thus $\pi^{(\mathrm{Env})}$ will stand for the canonical extension of $\pi$ to a representation of Env $(\mathfrak{g})$ on $\mathfrak{S}$ [with domain $D(\pi)$ ]. The representation $\pi$ is said to be symmetric (resp. skew-symmetric) if $\pi(g)$ is symmetric (resp. skew-symmetric) for all $g \in \mathfrak{g}$. The meaning of "irreducibility" and "(unitary) equivalence" of Lie algebra representations should be clear.

Given a net $\left(\mathfrak{g}_{2}\right)=\left(\operatorname{alg}\left(V, \mu_{\imath}\right)\right)$ of Lie algebras contracting into $\hat{g}=\operatorname{alg}(V, \hat{\mu})$ with respect to $\mathfrak{g}=\operatorname{alg}(V, \mu)$ by means of a reference net $\left(\Gamma_{\imath}\right)$ of automorphisms of $V$ and, for each $l \in J$, a representation $\pi_{l}$ of $\mathfrak{g}_{\imath}$ on a complex Hilbert space $\mathfrak{H}_{l}$, let $\tilde{\pi}_{l}$ be the representation $\pi_{l} \circ \Gamma_{\imath}^{-1}$ of $\mathfrak{g}$ on $\mathfrak{S}_{l}$. We put

$$
D_{J}\left(\pi_{l}\right)=\bigcap_{x \in \operatorname{Env}(\mathfrak{g})} D_{J}\left(\tilde{\pi}_{l}^{(\text {Env })}(x)\right)
$$

we denote by $D_{u}\left(\pi_{l}\right)$ the vector subspace of all $\left(\phi_{l}\right) \in D_{J}\left(\pi_{l}\right)$ such that we have

$$
\left[\tilde{\pi}_{l}^{(\mathrm{Env})}(x) \phi_{l}\right]=\left[\tilde{\pi}_{l}^{(\mathrm{Env})}(x) \phi_{l}^{\prime}\right]
$$


for all $x \in \operatorname{Env}(\mathfrak{g})$ whenever $\left(\phi_{l}^{\prime}\right)$ is an element of $D_{J}\left(\pi_{l}\right)$ in the equivalence class of $\left(\phi_{\imath}\right)$ modulo $\mathscr{J}$, and we write $D_{u}^{q}\left(\pi_{l}\right)$ instead of $\left[D_{u}\left(\pi_{\imath}\right)\right]$. Notice that

$$
D_{u}\left(\pi_{\imath}\right)=\bigcap_{x \in \operatorname{Env}(g)} D_{u}^{D_{J}\left(\pi_{\imath}\right)}\left(\tilde{\pi}_{t}^{(\text {Env })}(x)\right) .
$$

A net $\left(\pi_{\mathfrak{l}}\right)$ of representations of $\left(\mathfrak{g}_{\imath}\right)$ on $\left(\mathfrak{G}_{l}\right)$ is said to be contracting if $D_{u}\left(\pi_{\imath}\right)$ $=D_{J}\left(\pi_{l}\right) \neq\{0\}$. In other words, $\left(\pi_{l}\right)$ is contracting if $D_{J}\left(\pi_{l}\right) \neq\{0\}$ and

$$
D_{u}^{D_{J}\left(\pi_{\iota}\right)}\left(\tilde{\pi}_{i}^{(\mathrm{Env})}(x)\right)=D_{J}\left(\pi_{l}\right)
$$

for all $x \in \operatorname{Env}(\mathfrak{g})$. For each $g \in V$, we shall denote by $\pi_{J}(g)$ the operator defined by (II.2) with $A_{l}=\pi_{l}(g)$ and $F=D_{J}\left(\pi_{l}\right)$. Moreover, $\hat{\pi}_{J}$ will stand for the mapping of $V$ into the set of all operators in $\operatorname{cl}\left(D_{u}^{q}\left(\pi_{\imath}\right)\right)$ [the closure of $D_{u}^{q}\left(\pi_{\imath}\right)$ in $\mathfrak{H}_{J}$ ] obtained by restricting to $D_{u}^{q}\left(\pi_{l}\right)$ all the operators $\pi_{J}(g)$.

Remark 5. For each $g \in V$, each $\left(\phi_{l}\right) \in D_{u}\left(\pi_{l}\right)$, and each $\left(\phi_{l}^{\prime}\right) \in \mathscr{H}_{J}$, we have

$$
\left(\hat{\pi}_{J}(g)\left[\phi_{l}\right] \mid\left[\phi_{\imath}^{\prime}\right]\right)_{J}=\lim _{\imath}\left(\pi_{\imath}(g) \phi_{\imath} \mid \phi_{\imath}^{\prime}\right)_{l} .
$$

We can now realize as follows the proposal of Inönü and Wigner ([3], Sect. IIb) for the study of representations of contracted Lie algebras.

Proposition 3. Let $\left(\mathfrak{g}_{\imath}\right)=\left(\operatorname{alg}\left(V, \mu_{\imath}\right)\right)$ be a net of Lie algebras contracting into $\hat{\mathfrak{g}}=\operatorname{alg}(V, \hat{\mu})$ with respect to $\mathfrak{g}=\operatorname{alg}(V, \mu)$ by means of a reference net $\left(\Gamma_{\imath}\right)$ of automorphisms of $V$, and let $\left(\pi_{t}\right)$ be a contracting net of representations of $\left(\mathfrak{g}_{l}\right)$ on a net $\left(\mathfrak{G}_{l}\right)$ of complex Hilbert spaces. Then $\hat{\pi}_{J}$ is a representation of $\hat{\mathrm{g}}$ on $\mathrm{cl}\left(D_{u}^{q}\left(\pi_{l}\right)\right)$ with $\mathrm{D}\left(\hat{\pi}_{J}\right)=D_{u}^{q}\left(\pi_{l}\right)$.

Proof. The mapping $\hat{\pi}_{J}$ is obviously linear. Let $n$ be the dimension of $V$, let $\mathscr{G}=\left\{g_{j}\right\}_{1 \leqq j \leqq n}$ be a basis of $V$, and, for $1 \leqq j, k, l \leqq n$, let $\hat{c}_{j k}^{l}$ (resp. $c_{(t) j k}^{l}$ ) be the structure constants of $\hat{\mathfrak{g}}\left[\right.$ resp. $\left.\mathfrak{g}_{l}(l \in J)\right]$ with respect to $\mathscr{G}$. By Remark 4 , we have

$$
\begin{aligned}
& \left(\hat{\pi}_{J}\left(g_{j}\right) \hat{\pi}_{J}\left(g_{k}\right)-\hat{\pi}_{J}\left(g_{k}\right) \hat{\pi}_{J}\left(g_{j}\right)\right)\left[\phi_{l}\right] \\
& \quad=\left[\pi_{l}\left(\mu_{l}\left(g_{j}, g_{k}\right)\right) \phi_{l}\right]=\sum_{l=1}^{n}\left[c_{(l) j k}^{l} \pi_{l}\left(g_{l}\right) \phi_{l}\right]=\sum_{l=1}^{n} \hat{c}_{j k}^{l}\left[\pi_{l}\left(g_{l}\right) \phi_{l}\right] \\
& \quad=\hat{\pi}_{J}\left(\hat{\mu}\left(g_{j}, g_{k}\right)\right)\left[\phi_{l}\right]
\end{aligned}
$$

for all pairs $g_{j}, g_{k}$ of elements of $\mathscr{G}$ and all $\left[\phi_{l}\right] \in D_{u}^{q}\left(\pi_{l}\right)$.

The operation performed to obtain $\hat{\pi}_{J}$ is called a contraction (of a net of Lie algebra representations).

Let $\mathfrak{H}$ be a Hilbert space, let $\left(\mathfrak{H}_{l}\right)$ be a net of Hilbert spaces, and, for each $\imath \in J$, let $P_{\imath}$ be a continuous linear mapping of $\mathfrak{H}_{\text {into }} \mathfrak{H}_{l}$. The net $\left(\mathfrak{H}_{l}\right)$ is said to approximate $\mathfrak{S}$ with respect to the net $\left(P_{\imath}\right)([1]$, Sect. 2$)$ if $\left\|P_{\imath}\right\| \leqq 1$ for all $\iota \in J$ and $\lim _{\imath}\left\|P_{\imath} \phi\right\|_{\imath}=\|\phi\|_{\mathfrak{H}}$ for all $\phi \in \mathfrak{H}$. Then a net $\left(\phi_{\imath}\right) \in \prod \mathfrak{H}_{\imath}$ is $\left(P_{\imath}\right)$-convergent to $\phi \in \mathfrak{H}$, and we shall write $\phi=\left(P_{\imath}\right)-\lim \phi_{l}$, if $\lim _{l}\left\|\phi_{\imath}-P_{\imath} \phi\right\|_{\imath}=0$; a net $\left(A_{\imath}\right)$ of operators in $\left(\mathfrak{H}_{\imath}\right)$ is $\left(P_{\imath}\right)$-convergent to the operator $A$ in $\mathfrak{H}$, and we shall write $A=\left(P_{\imath}\right)-\lim A_{\imath}$, if $A \phi=\left(P_{\imath}\right)-\lim A_{\imath} P_{l} \phi$ for all $\phi \in \mathrm{D}(A)$. 
Proposition 4. Let $\left(\mathfrak{g}_{l}\right), \hat{\mathfrak{g}}, \mathfrak{g},\left(\Gamma_{\imath}\right)$ be as in Proposition 3 and let $\mathscr{G}$ be a basis of $V$. For each $\imath \in J$, let $\mathfrak{Y}_{l}$ be a complex Hilbert space of dimension at most Card $(\mathbf{R})$, let $\pi_{\imath}$ be a skew-symmetric representation of $\mathfrak{g}_{l}$ on $\mathfrak{H}_{l}$, let $S_{l}$ be a subset of $\mathbf{R}$ such that $S_{l^{\prime}} \leqq S_{l}$ whenever $\imath^{\prime}<l$, and let $\left\{\phi_{l}^{(s)}\right\}_{s \in S_{l}}$ be an orthonormal basis of $\mathfrak{H}_{\imath}$ contained in $\mathrm{D}\left(\pi_{l}\right)$. Suppose that for $-k \leqq m \leqq k$, where $k$ is a fixed positive integer, for each $s \in S=\bigcup_{l} S_{\imath}$, and each $g \in \mathscr{G}$, we have a net $\left(c_{\imath, s, m}(g)\right)$ of complex numbers, which are 0 whenever $s \notin S_{\imath}$ or $s+m \notin S_{\imath}$, converging in $\mathbf{C}$ to $c_{s, m}(g)$. If

$$
\pi_{l}(g) \phi_{l}^{(s)}=\sum_{m=-k}^{k} c_{l, s, m}(g) \phi_{l}^{(s+m)}
$$

for all $\imath \in J$, all $s \in S_{\imath}$, all $g \in \mathscr{G}$, and if there exist two real numbers $v(g), t(g)$ such that

$$
\left|c_{s, m}(g)\right| \leqq v(g)(|s|+|t(g)|)
$$

for $-k \leqq m \leqq k$, all $s \in S$, and all $g \in \mathscr{G}$, then

(i) For each $s \in S$, the net $\left(\psi_{\imath}^{(s)}\right) \in \prod_{l} \mathfrak{H}_{l}$, where $\psi_{l}^{(s)}=\phi_{l}^{(s)}$ whenever $s \in S_{\imath}$ and $\psi_{\imath}^{(s)}=0$ otherwise, is convergent and $\mathfrak{\Xi}_{J}=\left\{\left[\psi_{\imath}^{(s)}\right]\right\}_{s \in S}$ is an orthonormal system in $\mathfrak{S}_{J}$.

(ii) $\hat{\pi}_{J}$ is a skew-symmetric representation of $\hat{\mathrm{g}}$ on $\operatorname{cl}\left(D_{u}^{q}\left(\pi_{l}\right)\right)$ with $\mathrm{D}\left(\hat{\pi}_{J}\right)=D_{u}^{q}\left(\pi_{l}\right)$.

(iii) For each $g \in \mathscr{G}, \mathrm{sp}\left(\mathfrak{S}_{J}\right)$ is a $\hat{\pi}_{J}(\hat{\mathrm{g}})$-stable set of analytic vectors for $\hat{\pi}_{J}(g)$.

(iv) The restriction $\hat{\pi}$ of $\hat{\pi}_{J}$ to $\mathrm{sp}\left(\mathfrak{S}_{J}\right)$ is a skew-symmetric representation of $\hat{\mathfrak{g}}$ on the closure $\mathfrak{H}$ of $\operatorname{sp}\left(\mathfrak{S}_{J}\right)$ in $\mathfrak{H}_{J}$; for each $g \in \mathscr{G}$ and each $\mathrm{S} \in S$, we have

$$
\hat{\pi}(g)\left[\psi_{l}^{(s)}\right]=\sum_{m=-k}^{k} c_{s, m}(g)\left[\psi_{l}^{(s+m)}\right] .
$$

(v) The net $\left(\mathfrak{G}_{l}\right)$ approximates $\mathfrak{H}$ with respect to the net $\left(P_{\imath}\right)$ defined in $\mathfrak{G}_{J}$ by $P_{l}\left[\psi_{l^{\prime}}^{(s)}\right]=\psi_{l}^{(s)}(s \in S)$ and extended to $\mathfrak{H}$ by linearity and continuity. For each $g \in V$, we have $\hat{\pi}(g)=\left(P_{l}\right)-\lim \pi_{l}(g)$.

Proof. Assertion (i) follows at once from the remark that, by reason of the assumption on the sets $S_{l}(l \in J)$ we have $\left(\psi_{l}^{(s)}\right) \in \mathscr{H}_{J}$ and $\left[\psi_{l}^{(s)}\right] \neq 0$ for all $s \in S$. Now for each $g \in V$ and each $n \in \mathbf{N}^{*}$, we have

$$
D_{u}^{D_{J}\left(\pi_{l}\right)}\left(\pi_{l}(g)^{n}\right)=D_{J}\left(\pi_{l}\right)
$$

by virtue of Proposition 1 ; it follows, on account of Remark 4 and by using the Poincaré-Birkhoff-Witt theorem [7], that $D_{u}\left(\pi_{t}\right)=D_{J}\left(\pi_{t}\right)$. On the other hand, $\operatorname{sp}\left(\left\{\left(\psi_{l}^{(s)}\right)\right\}_{s \in S}\right) \subseteq D_{J}\left(\pi_{l}\right)$ by (II.5), so that the net $\left(\pi_{l}\right)$ is contracting. Then Proposition 3 implies (ii), the skew-symmetry of $\hat{\pi}_{J}$ being a consequence of that of the $\pi_{l}(l \in J)$.

Assertion (iii) follows from Proposition 2, whereas (iv) is obvious. To prove (v) it is sufficient to note that, for each $g \in \mathscr{G}$ and each $s \in S$, we have

$$
P_{\imath} \hat{\pi}(g)\left[\psi_{l^{\prime}}^{(s)}\right]=\sum_{m=-k}^{k} c_{s, m}(g) \psi_{l}^{(s+m)},
$$

whence

$$
\begin{aligned}
& \lim _{\imath}\left\|\pi_{\imath}(g) P_{\imath}\left[\psi_{\imath^{\prime}}^{(s)}\right]-P_{\imath} \hat{\pi}(g)\left[\psi_{\imath^{\prime}}^{(s)}\right]\right\|_{\imath} \\
& \leqq \sum_{m=-k}^{k} \lim _{\imath}\left|c_{\imath, s, m}(g)-c_{s, m}(g)\right|=0 .
\end{aligned}
$$


Remark 6. By virtue of Proposition 1, Proposition 4 is still true if "skewsymmetric" is everywhere replaced by "symmetric".

Remark 7. Since the topological space $\mathfrak{M}_{V}$ is metrizable, it is always possible to use countable index sets in the study of Lie algebra contractions. But this is, a priori, no longer the case when contractions of representations are considered.

Let $G$ be a finite-dimensional real Lie group, let Lie $(G)$ be its Lie algebra, and let $\mathfrak{H}$ be a complex Hilbert space. We remind that a strongly continuous unitary representation $U$ of $G$ on $\mathfrak{H}$ defines a skew-symmetric representation $d U$ of Lie $(G)$ on $\mathfrak{H}$ by

$$
d U(g) \phi=\lim _{t \rightarrow 0} \frac{U(\exp (t g)) \phi-\phi}{t} \quad\left(\phi \in \mathfrak{H}^{\infty}(G)\right),
$$

where $\mathfrak{H}^{\infty}(G)=\mathrm{D}(d U)$ is the vector subspace of all $\phi \in \mathfrak{H}$ such that the mapping $x \mapsto U(x) \phi$ of $G$ into $\mathfrak{H}$ is of class $C^{\infty}$. The representation $d U$ is called the differential of $U$. A skew-symmetric representation $\pi$ on $\mathfrak{H}$ of a finite-dimensional real Lie algebra $g$ is said to be integrable if, for every simply connected Lie group $G$ whose Lie algebra is isomorphic to $\mathfrak{g}$, there exist a (necessarily unique) strongly continuous unitary representation $U$ of $G$ on $\mathfrak{H}$ and an isomorphism $\theta$ of $\mathfrak{g}$ onto Lie $(G)$ such that $\pi(g) \cong d U(\theta(g))$ for all $g \in \mathfrak{g}$.

By ([8], Theorem 1), we now have the

Corollary. Let $G$ be a simply connected finite-dimensional real Lie group and let $\mathfrak{g}=\operatorname{alg}(V, \mu)$ be its Lie algebra. For each $t \in J$, let $U_{\imath}$ be a strongly continuous unitary representation of $G$ on a complex Hilbert space $\mathfrak{H}_{1}$ of dimension at most Card $(\mathbf{R})$, let $\left(\Gamma_{\imath}\right)$ be a reference net of automorphisms of $V$ for a contraction $\hat{\mathfrak{g}}$ of $\mathfrak{g}$, and put $\pi_{\imath}=d U_{\imath} \circ \Gamma_{l}$. If $\mathscr{G}_{,} S_{l},\left\{\phi_{l}^{(s)}\right\}_{s \in S_{\imath}}$, and $\left(c_{\imath, s, m}(g)\right)$ are as in Proposition 4 and if (II.5), (II.6) are satisfied, then the skew-symmetric representation $\hat{\pi}$ of $\hat{\mathfrak{g}}$ on $\mathfrak{H}$ defined in Proposition 4(iv) is integrable.

\section{References}

1. Trotter, H.F.: Approximation of semi-groups of operators. Pacific J. Math. 8, 887-919 (1958)

2. Segal, I.E. : A class of operator algebras which are determined by groups. Duke Math. J. 18, 221-265 (1951)

3. Inönü, E., Wigner, E.P.: On the contraction of groups and their representations. Proc. Natl. Acad. Sci. U.S.A. 39, 510-524 (1953)

4. Saletan, E.J.: Contraction of Lie groups. J. Math. Phys. 2, 1-21 (1961)

5. Lévy-Nahas, M.: Deformation and contraction of Lie algebras. J. Math. Phys. 8, 1211-1222 (1967)

6. Kurtz, T.G. : Extensions of Trotter's operator semigroup approximation theorems. J. Funct. Anal. 3, 354-375 (1969)

7. Jacobson, N.: Lie algebras. New York: Interscience 1962

8. Flato, M., Simon, J., Snellman, H., Sternheimer, D.: Simple facts about analytic vectors and integrability. Ann. Sci. Éc. Norm. Sup. (4ème série) 5, 423-434 (1972) 